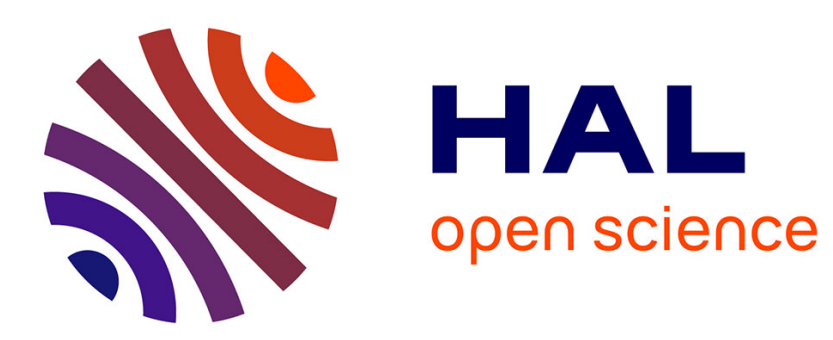

\title{
STAT6 is a cargo of exportin 1: Biological relevance in primary mediastinal B-cell lymphoma
}

Hadjer Miloudi, Karen Leroy, Fabrice Jardin, Brigitte Sola

\section{To cite this version:}

Hadjer Miloudi, Karen Leroy, Fabrice Jardin, Brigitte Sola. STAT6 is a cargo of exportin 1: Biological relevance in primary mediastinal B-cell lymphoma. Cellular Signalling, 2018, 46, pp.76-82. 10.1016/j.cellsig.2018.02.016 . hal-01725066

\section{HAL Id: hal-01725066 \\ https://hal-normandie-univ.archives-ouvertes.fr/hal-01725066}

Submitted on 7 Mar 2018

HAL is a multi-disciplinary open access archive for the deposit and dissemination of scientific research documents, whether they are published or not. The documents may come from teaching and research institutions in France or abroad, or from public or private research centers.
L'archive ouverte pluridisciplinaire HAL, est destinée au dépôt et à la diffusion de documents scientifiques de niveau recherche, publiés ou non, émanant des établissements d'enseignement et de recherche français ou étrangers, des laboratoires publics ou privés. 


\section{Accepted Manuscript}

STAT6 is a cargo of exportin 1: Biological relevance in primary mediastinal B-cell lymphoma

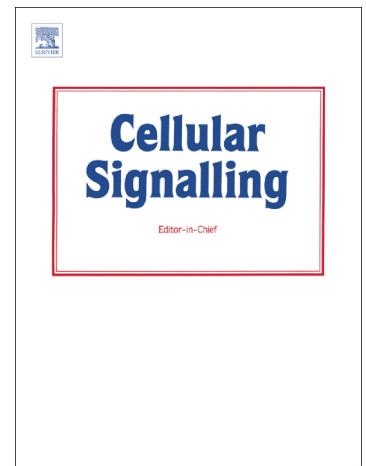

Hadjer Miloudi, Karen Leroy, Fabrice Jardin, Brigitte Sola

PII: $\quad$ S0898-6568(18)30063-9

DOI: $\quad$ doi:10.1016/j.cellsig.2018.02.016

Reference: $\quad$ CLS 9085

To appear in: Cellular Signalling

Received date: $\quad 1$ February 2018

Revised date: $\quad 26$ February 2018

Accepted date: $\quad 28$ February 2018

Please cite this article as: Hadjer Miloudi, Karen Leroy, Fabrice Jardin, Brigitte Sola , STAT6 is a cargo of exportin 1: Biological relevance in primary mediastinal B-cell lymphoma. The address for the corresponding author was captured as affiliation for all authors. Please check if appropriate. Cls(2017), doi:10.1016/j.cellsig.2018.02.016

This is a PDF file of an unedited manuscript that has been accepted for publication. As a service to our customers we are providing this early version of the manuscript. The manuscript will undergo copyediting, typesetting, and review of the resulting proof before it is published in its final form. Please note that during the production process errors may be discovered which could affect the content, and all legal disclaimers that apply to the journal pertain. 


\section{STAT6 is a cargo of exportin 1: biological relevance in primary}

\section{mediastinal B-cell lymphoma}

Hadjer Miloudi ${ }^{1}$, Karen Leroy ${ }^{2}$, Fabrice Jardin ${ }^{3,4}$, Brigitte Sola ${ }^{1,{ }^{*}}$

${ }^{1}$ Normandie Univ, INSERM UMR1245, UNICAEN, Caen, France

${ }^{2}$ Université Paris-Descartes, Paris, France

${ }^{3}$ Département d'Hématologie, Centre Henri Becquerel, Rouen, France

${ }^{4}$ Normandie Univ, INSERM UMR1245, UNIROUEN, Rouen, France

* Corresponding author. E-mail: Brigitte Sola, brigitte.sola@unicaen.fr

Authors E-mail: hadjer.miloudi@unicaen.fr; karen.leroy@aphp.fr;

fabrice.jardin@chb.unicancer.fr 


\section{Abstract}

Primary mediastinal B-cell lymphoma (PMBL) is a distinct B-cell lymphoma subtype with unique clinicopathological and molecular features. PMBL cells are characterised by several genetic abnormalities that conduct to the constitutive activation of the Janus kinase 2 /signal transducer and activator of transcription 6 (JAK2/STAT6) signalling pathway. Among recurrent genetic changes in $\mathrm{PMBL}$, we previously reported that the $X P O 1$ gene encoding exportin 1 that controls the nuclear export of cargo proteins and RNAs, is mutated (p.E571K) in about $25 \%$ of PMBL cases. We therefore hypothesized that STAT6 could be a cargo of XPO1 and that STAT6 cytoplasm/nucleus shuttle could be altered in a subset of PMBL cells. Using immunocytochemistry techniques as well as the proximity ligation assay, we showed that STAT6 bound XPO1 in PBML cell lines and in HEK-293 cells genetically engineered to produce STAT6. Moreover, XPO1-mediated export of STAT6 occurs in cells expressing either a wild-type or the E571K mutated XPO1 protein.

Keywords : B lymphoma, CRM1, SINE, STAT, PLA technology, XPO1 mutation

Abbreviations : DAPI, 4',6-diamidino-2-phénylindole ; DMSO, dimethylsulfoxide ; FCS, foetal calf serum; HSP, heat shock protein; IB, immunoblotting; IF, immunofluorescence; IL, interleukin; JAK, Janus kinase; NES, nuclear export signal; NLS, nuclear localisation signal; p-, phosphorylated; PARP, poly(ADP-ribose) polymerase; PLA, proximity ligation assay; PMBL, primary mediastinal B-cell lymphoma; STAT, signal transducer and activator of transcription; u-, unphosphorylated; XPO1, exportin 1 


\section{Introduction}

Primary mediastinal B-cell lymphoma (PMBL) is a subtype of diffuse large B-cell lymphoma (DLBCL) which displays recurrent alterations of the Janus kinase-signal transducer and activator of transcription (JAK-STAT) survival pathway leading to its constitutive activation [1]. Moreover, in almost 35\% of the cases, PMBL cells harbour mutations within the DNA binding domain of STAT6 [2]. The functional consequences of such mutations are still unknown in PMBL [3] but are supposed to be activating in follicular lymphoma [4]. PMBL cells exhibit also a recurrent (25\% of the cases) mutation of the XPO1 (or CRM1) gene [5]. This gene encodes exportin 1 (XPO1), a member of the karyopherin $\beta$ family, which mediates the nuclear export of various RNAs and cargo proteins possessing a leucine-rich nuclear export signal (NES) [6]. Several transcription factors are cargos of XPO1 including STAT1 [7] and STAT3 [8] among the STAT family. Given the crucial role of STAT6 and XPO1 proteins in the physiopathology of the disease and their potential as targets for personalized medicine $[3,5]$, we thought to identify STAT6 as a new cargo for XPO1. Moreover, we analysed the potential effects of XPO1/STAT6 mutations on STAT6 export.

\section{Materials and Methods}

\subsection{Cells culture}

The HEK-293 STAT6-deficient cell line was stably transfected with an expression vector coding for STAT6 and hereafter referred to as 293-S6 [2]. The PMBL cell lines MedB1, Karpas 1106 (hereafter referred to as K1106), and U2940 have been described elsewhere [5]. PMBL cells harbour mutations of XPO1 or/and STAT6 (Table 1). Cell lines were cultured in RPMI 1640 medium (Lonza) supplemented with 10-20\% foetal calf serum (FCS, PAA Laboratories), 
$2 \mathrm{mM} \mathrm{L-glutamine}$ and antibiotics (Lonza), under a humid atmosphere at $37^{\circ} \mathrm{C}$. $293-\mathrm{S} 6$ cells were maintained in DMEM medium (Lonza) supplemented with $10 \%$ FCS, L-glutamine, geneticin (500 $\mathrm{\mu g} / \mathrm{ml}$, Gibco) and antibiotics. In some experiments, cells were incubated with $1 \mu \mathrm{M}$ KPT-330 (Selinexor, Sigma-Aldrich) for $4 \mathrm{~h}$.

\subsection{Indirect immunofluorescence and confocal microscopy analysis}

For the indirect immunofluorescence (IF) analyses, 293-S6 cells were grown directly onto poly-L-lysine-coated coverslips. Cultured PMBL cells were cytospun on Superfrost glass slides. Cells were then fixed in $4 \%$ paraformaldehyde and permeabilised in $0.5 \%$ Triton-X100. The slides were then stained with primary antibodies (Abs), and then with Alexa Fluor 488(in red) or Alexa Fluor 546- (in green) conjugated goat anti-mouse or -rabbit IgG, respectively, and counterstained with DAPI (4',6-diamidino-2-phenylindole dihydrochloride, Molecular Probes, in blue). Primary Abs against XPO1 (sc-74454) and RanBP1 (ab97659) were purchased from Santa Cruz Biotechnologies and abcam, respectively. Abs against STAT6 (HPA001861) and p-Tyr641-STAT6 (SAB4504546) were purchased from Sigma-Aldrich. Slides were observed with a confocal fluorescence microscope (Fluoview FV 100, Olympus). The colocalisation of XPO1 and STAT6 proteins was investigated with the ImageJ software as described previously [9].

\subsection{Proximity ligation assay}

The proximity ligation assay (PLA) technology [10] was used to detect in situ XPO1-STAT6 proteins interaction. We used the Duolink ${ }^{\circledR}$ In Situ Red Starter Kit (DU092101, SigmaAldrich) according to the supplier's instructions. Cells were fixed, permeabilised, and incubated with the primary Abs as described for IF. Duolink ${ }^{\circledR}$ secondary Abs were then 
added. These secondary Abs are conjugated to oligonucleotides able to form a closed circle via base pairing and ligation using the Duolink ligation solution. This occurs only when the Abs are in close proximity (less than $40 \mathrm{~nm}$ ). The detection of signals is conducted by rolling circle amplification using DNA polymerase incorporating fluorescent labelled nucleotides into the amplification products. The resulting positive signals are visualized as red fluorescent dots; each dot represents one interaction event. We used an Ab against XPO1 (sc-74454) and as a positive control, an anti-RanBP1 (ab97659, abcam), RanBP1 being a wellknown partner of XPO1 [11]. As a negative control, no primary Ab was added in the reaction mixture. Slides were observed with a confocal fluorescence microscope (Fluoview FV 100, Olympus).

\subsection{Proliferation assay}

PMBL cells were seeded in 96-well plates in complete medium $\left(2 \times 10^{5}\right.$ cells/200 $\left.\mu \mathrm{l}\right)$ and treated for $48 \mathrm{~h}$ with vehicle (0.01\% dimethylsulfoxide, DMSO) or various concentrations of KPT-330 (selinexor, 0.1-20 nM). Cell viability was quantified using an MTS assay (CellTiter 96® $\mathrm{AQ}_{\text {ueous }}$ One Solution Cell Proliferation Assay, Promega) according to the manufacturer's instructions.

\subsection{Proteins purification and immunoblotting}

Cytoplasmic and nuclear extracts were prepared with the Cell fractionation kit (\#9038, Cell Signaling Technology) from cultured cells. The purity of extracts was checked by immunoblotting (IB) with Abs directed against the strictly nuclear poly-(ADP-ribose) polymerase protein (PARP, clone 4F10, sc-69703, Santa Cruz Biotech.), and the heat shock protein (HSP) 90 cytosolic protein (clone F-2, sc-8007, Santa Cruz Biotech.). The method used 
for IB has been described elsewhere [12]. Abs against STAT6 (\#9362) and p-Tyr641-STAT6 (\#9361) were purchased from Cell Signaling Technology.

\section{Results}

\subsection{The JAK2/STAT6 pathway is constitutively activated in PMBL cell lines}

We previously stably transfected the HEK-293 STAT6-deficient fibroblastic cell line with an expression vector coding for STAT6 [2]. The stimulation of 293-S6 cells with interleukin 4 (IL4) led to the activation of the JAK-STAT6 pathway and the phosphorylation of STAT6 on Tyr641 residues (Fig. 1a). As soon as 30 min after IL4 stimulation, the phosphorylated (p-) form of STAT6 was detected in the nucleus and the cytoplasm of 293-S6 cells. It has been described that STAT6 shuttles between the cytoplasm and the nucleus continually. However, only the phosphorylated form accumulates in the nucleus as the result of its ability to bind DNA [13]. This was observed in IL4-stimulated 293-S6 cells. Indeed, the level of p-Tyr641STAT6 remained high in the nucleus and decreased in the cytoplasm between $4 \mathrm{~h}$ - and $6 \mathrm{~h}$ post-treatment.

The localization of STAT6 and p-Tyr641-STAT6 was next assessed by IF studies for IL4stimulated/unstimulated 293-S6 cells and unstimulated PBML cells (Fig. 1b, c). In unstimulated 293 -S6 cells, a typical STAT6 cytoplasmic immunostaining was observed. After IL4-stimulation, STAT6 was both cytoplasmic and nuclear, and its phosphorylated form was mainly nuclear (Fig. 1b). In the three PMBL cell lines analysed, STAT6 was localised both in the cytoplasmic and nuclear compartments, and p-STAT6 was mainly nuclear (Fig. 1c). We confirmed that the JAK2/STAT6 pathway is constitutively activated in PMBL cells and that STAT6 shuttles between the cytoplasm and the nucleus. Moreover, In PMBL cells, this occurred whatever the mutated/unmutated XPO1 and STAT6 status (Table 1). 


\subsection{A NES signal is present in human STAT6}

Because the structure of STAT family members is highly conserved among the proteins and along the evolution [14], we suspected that STAT6 could possess a NES. We analysed the protein sequence with LocNes, a computational tool for locating NES in proteins [15]. As shown Table 2, STAT6 displayed two NES putative binding sites that fit with the consensus sequences defined by $\mathrm{Xu}$ and colleagues [16].

\subsection{XPO1 and STAT6 colocalise in 293-S6 and PMBL cells}

The colocalisation of STAT6 and XPO1 in 293-S6 was analysed by indirect confocal IF and image analysis. As shown Fig. 2a, XPO1 and STAT6 colocalised in 293-S6 cells. Indeed, an orange staining appeared when the red XPO1 staining and the green STAT6 staining were merged. This was confirmed when images were processed with the ImageJ software. When the fluorescence intensity of each fluorophore was plotted along a line crossing one representative cell, the red and the green signals peaked together in the nuclear compartment (Fig. 2a). Moreover, cytoplasmic STAT6 was free of XPO1 in agreement with the mechanism of XPO1-mediated export. Indeed, once in the cytoplasm, XPO1/cargos dimers disassemble and XPO1 shuttles back to the nucleus [6].

We next analysed the localization of XPO1 and STAT6 in PBML cell lines harbouring (MedB1) or not (U2940) a mutated XPO1 gene with the same tools. Whatever the XPO1 status, we found that STAT6 and XPO1 colocalised in the nuclear compartment (Fig. 2b). Free STAT6 was detected in the cytoplasm (Fig. 2b, black box) and XPO1 accumulated mainly at perinuclear sites, again in agreement with the export mechanism [17]. 


\subsection{XPO1 and STAT6 interact in 293-S6 and PMBL cells}

We further used the DUOLink ${ }^{\circledR}$ PLA technology for identifying in situ interactions of XPO1 and STAT6. As shown Fig. 3, in the negative control, no red fluorescent signal was detected. In the positive control, each interaction of XPO1 with RanBP1 was revealed by a single red dot. We observed such interactions of XPO1 with STAT6 in the 293-S6 cells and in the two PMBL cell lines tested despite the presence of the XPO1 E571K and STAT6 N417Y/N430T mutations in MedB1 cells.

\subsection{STAT6 is a cargo of XPO1}

KPT-330 (or Selinexor) is a selective inhibitor of nuclear export (SINE) that inhibits XPO1 function by binding to its hydrophobic groove on the cysteine 528 [17]. XPO1 inhibition leads to the accumulation of cargo proteins within the nucleus. We first explored the sensitivity of PMBL cell lines to Selinexor with the MTS proliferation assay. As shown Fig. 4a, the three cell lines are sensitive to Selinexor and exhibited similar sensitivities (see the calculated IC $C_{50}$ ). Moreover, in keeping with previous results [5], mutated and unmutated XPO1 proteins were similarly inhibited in Selinexor-treated PMBL cell lines. As expected, Selinexor induced RanBP1 (as a control) and STAT6 sequestration into the nucleus in both K1106 and MedB1 cell lines (Fig. 4b).

\section{Discussion}

STAT6 belongs to the STAT family of both adaptor proteins and transcription factors. STAT family members display a similar protein structure that is instrumental for their activation and functions. Moreover, they possess regulatory signals that ensure their distribution to the right cellular location at the right time. The canonical signalling is initiated by the binding 
of an extracellular ligand to its cognate transmembrane receptor. This binding induces the dimerization and activation of JAKs. Activated JAKs phosphorylate tyrosine residues on the cytoplasmic part of the receptor that serve as docking sites for STAT factors. Phosphorylated STATs dimerize and translocate to the nucleus to activate or repress their target genes. However, non-canonical JAK/STAT pathways exist and play a pivotal role in heterochromatin maintenance and tumour suppression $[18,19]$. In particular, nuclear unphosphorylated (u-) STAT1, STAT3 and STAT5A proteins are capable to activate a subset of specific target genes. The JAK2/STAT6 signalling pathway is a survival pathway involved in the proliferation and differentiation of B cells. Deregulated JAK2/STAT6 signalling participates the pathogenesis of subtypes of lymphoma [20]. In PMBL cells, the presence of nuclear phosphorylated STAT6 indicates a constitutive activated JAK2/STAT6 signalling pathway [21]. This can be the consequence of an amplification of the JAK2 gene [22], recurrent mutations of SOCS1 or PTPN1 genes [23-25]. In agreement with previous studies, we observed that $p$-Tyr641-STAT6 is present in the nucleus of unstimulated PMBL cells confirming that the JAK2/STAT6 pathway is constitutively activated. However, despite this continuous activation, STAT6 shuttles between the nucleus and the cytoplasm suggesting that import/export mechanisms are correctly regulated.

XPO1 mediates the nuclear export of more than 200 proteins [6]. We recently identified a recurrent mutation of XPO1 gene (p.E571K) in $25 \%$ of PMBL or Hodgkin lymphoma patients $[5,26]$. The mutation is located close to the hydrophobic groove of XPO1 which is responsible of cargo recognition. The functional consequences of this mutation are still unknown, but could influence the interactions of XPO1 with a subset of cargo proteins. Indeed, the change from a negatively charged (glutamate) to a positively charged (lysine) residue could modify the affinity of XPO1 for NESs bearing negative charges [27]. 
Alternatively, XPO1 mutation could have no effect on cargos recognition and binding and be involved in other biological function such as spindle formation control. Here we demonstrated that XPO1 and STAT6 colocalise in the nucleus and bound each other in PMBL cell lines. Moreover, the nuclear export of STAT6 is inhibited by Selinexor, confirming that STAT6 is a cargo of XPO1 even in MedB1 cells harbouring both STAT6 and XPO1 mutations. However, both wt and mutated XPO1 alleles are transcribed in MedB1 cells (data not shown). Although, we cannot exclude formally that only the wild-type fraction of the XPO1 protein is fully capable of export, this hypothesis seems unlikely. Indeed, using the PLA technology, we observed the same number of spots, indicative of XPO1-cargos interactions, in the mutated vs. unmutated XPO1-harbouring cell lines and for RanBP1 and STAT6 proteins.

Regarding STATs signalization from the cell surface to the nucleus, their distribution inside the cell compartments must be tightly regulated. The most obvious regulation for STATs localization is the recognition of NLS (nuclear localization sequence) for nuclear import and NES for nuclear export by importin/exportin, respectively. However, two mechanisms regulate STAT3 and STAT1 transport into the nucleus $[7,8]$. The first one is the canonical ligand-dependent activation pathway and the second is active in unstimulated quiescent cells. This last mechanism allows the nuclear import u-STAT3 and -STAT1. The regulation of STAT3 nuclear export is under the control of three NESs, two of them (404-414 and 524-535) regulate the "basal" nuclear export for U-STAT3, the third one (306-318) is implicated in the post-stimulation canonical response. Using the LocNES computational tool, we were able to identify two potential NESs in STAT6. The first NES is located within the coiled-coil domain of STAT6 at the same position than the NES of STAT1 necessary for nuclear export [28]. The other one is located in the DNA binding domain, at the same position than the second NES of 
STAT3 involved in the basal response [8] (Fig. 5). Thus, we can speculate that the first STAT6 NES is involved in the ligand-dependent response whereas the second one is ligandindependent. In agreement with this statement, Chen and colleagues showed that u-STAT6 can continuously shuttle between the nucleus and the cytoplasm [13]. Since u-STAT and pSTAT proteins may target different sets of genes coding for proteins involved in distinct cell processes [29], further studies are necessary to confirm this point for PMBL cells.

\section{Conclusions}

We report in this study that STAT6 is a cargo of XPO1 in normal (293-S6) and tumour (PMBL) cells. STAT6 recognition and binding occur in cells having a constitutive activation of the JAK2/STAT6 pathway (PMBL cells) and in IL4-stimulated cells (293-S6). Although PMBL cells display STAT6 and/or XPO1 genetic alterations (they are not mutually exclusive), both proteins maintain their cellular functions. This is particularly important since both STAT6 and XPO1 proteins are potential targets for PBML therapies $[3,5]$. The additive/synergistic efficacy of SINEs together with JAK2 inhibitors (unless STAT6 inhibitors are available) should be tested in preclinical models. Our findings highlight novel therapeutic potentials for PMBL disease.

Declarations of interest : none

\section{Funding resources}

This work was partially financially supported by the GEFLUC Rouen - Normandie (to BS).

\section{Acknowledgements}


The authors thank Anne Barbaras (UNICAEN) for help with cell culture, Fabrice Gouilleux

(LNox, CNRS, Université François Rabelais, Tours) for helpful discussions, ICORE (Université de (aen) for microscopy facilities.

\section{Author Contributions}

Conceptualization: HM, KL, FJ, BS ; formal analysis: HM ; funding acquisition: BS ;

methodology: $\mathrm{HM}$; resources: $\mathrm{KL}, \mathrm{FJ}$; supervision: $\mathrm{BS}$; validation: $\mathrm{FJ}, \mathrm{BS}$; writing original draft: $\mathrm{HM}, \mathrm{BS}$; review and editing: $\mathrm{HM}, \mathrm{KL}, \mathrm{FJ}, \mathrm{BS}$.

\section{References}

[1] C. Steidl, R.D. Gascoyne, The molecular pathogenesis of primary mediastinal large Bcell lymphoma, Blood. 118 (2011) 2659-2669. doi:10.1182/blood-2011-05-326538.

[2] O. Ritz, C. Guiter, F. Castellano, K. Dorsch, J. Melzner, J.P. Jais, et al., Recurrent mutations of the STAT6 DNA binding domain in primary mediastinal B-cell lymphoma, Blood. 114 (2009) 1236-1242. doi:10.1182/blood-2009-03-209759.

[3] K. Leroy, A. Pujals, L. Pelletier, Targeting STAT6 in PMBL, Oncotarget. 5 (2014) 7216.

[4] M. Yildiz, H. Li, D. Bernard, N.A. Amin, P. Ouillette, S. Jones, et al., Activating STAT6 mutations in follicular lymphoma, Blood. 125 (2014) 668-679. doi:10.1182/blood2014.

[5] F. Jardin, A. Pujals, L. Pelletier, E. Bohers, V. Camus, S. Mareschal, et al., Recurrent mutations of the exportin 1 gene (XPO1) and their impact on selective inhibitor of nuclear export compounds sensitivity in primary mediastinal B-cell lymphoma, Am J Hematol. 91 (2016) 923-930. doi:10.1002/ajh.24451.

[6] V. Camus, H. Miloudi, A. Taly, B. Sola, F. Jardin, XPO1 in B cell hematological malignancies: from recurrent somatic mutations to targeted therapy, (2017) 1-13. doi:10.1186/s13045-017-0412-4.

[7] K.M. McBride, N.C. Reich, The Ins and Outs of STAT1 Nuclear Transport, Science Signaling. 195 (2003) re13.

[8] S. Bhattacharya, C. Schindler, Regulation of STAT3 nuclear export, J Clin Invest. 111 (2003) 553-559.

[9] L. Xu, G. Li, J. Wang, Y. Fan, Z. Wan, S. Zhang, et al., Through an ITIM-independent mechanism the FcyRIIB blocks $B$ cell activation by disrupting the colocalized microclustering of the B cell receptor and CD19, J Immunol. 192 (2014) 5179-5191. doi:10.4049/jimmunol.1400101.

[10] S. Fredriksson, M. Gullberg, J. Jarvius, C. Olsson, K. Pietras, S.M. Gústafsdóttir, et al., Protein detection using proximity-dependent DNA ligation assays, Nature Biotechnology. 20 (2002) 473-477.

[11] H.Y. Fung, Y.M. Chook, Atomic basis of CRM1-cargor recognition, release and inhibition. Semin Cancer Biol. 27 (2014) 52-61. 
doi:10.1016/j.semcancer.2014.03.002.

[12] S. Body, A. Esteve-Arenys, H. Miloudi, C. Recasens-Zorzo, G. Tchakarska, A. Moros, et al., Cytoplasmic cyclin D1 controlsthe migration and invasiveness ofmantle lymphoma cells, Scientific Rep. (2017) 1-12. doi:10.1038/s41598-017-14222-1.

[13] H.C. Chen, N.C. Reich, Live cell imaging reveals continuous STAT6 nuclear trafficking, J Immunol. 185 (2010) 64-70. doi:10.4049/jimmunol.0903323.

[14] W.X. Li, Canonical and non-canonical JAK-STAT signaling, Trends in Cell Biology. 18 (2008) 545-551. doi:10.1016/j.tcb.2008.08.008.

[15] D. Xu, K. Marquis, J. Pei, S.-C. Fu, T. Cağatay, Nick V Grishin, et al., LocNES: a computational tool for locating classical NESs in CRM1 cargo proteins, Bioinformatics. 31 (2015) 1357-1365. doi:10.1093/bioinformatics/btu826/-/DC1.

[16] D. Xu, A. Farmer, G. Collett, N.V. Grishin, Y.M. Chook, Sequence and structural analyses of nuclear export signals in the NESdb database, Mol Biol Cell. 23 (2012) 3677-3693. doi:10.1091/mbc.E12-01-0046.

[17] J.E. Neggers, T. Vercruysse, M. Jacquemyn, E. Vanstreels, E. Baloglu, S. Shacham, et al., Identifying drug-target selectivity of small-molecule CRM1/XPO1 inhibitors by CRISPR/Cas9 genome editing, Chem Biol. 22 (2015) 107-116. doi:10.1016/j.chembiol.2014.11.015.

[18] X. Hu, P. Dutta, A. Tsurumi, J. Li, J. Wang, H. Land, et al., Unphosphorylated STAT5A stabilizes heterochromatin and suppresses tumor growth, Proc Natl Acad Sci USA. 110 (2013) 10213-10218. doi:10.1073/pnas.1221243110.

[19] A. Tsurumi, C. Zhao, W.X. Li, Canonical and non-canonical JAK/STAT transcriptional targets may be involved in distinct and overlapping cellular processes, BMC Genomics (2017) 1-10. doi:10.1186/s12864-017-4058-y.

[20] L.M. Scott, M.K. Gandhi, Deregulated JAK/STAT signalling in lymphomagenesis, and its implications for the development of new targeted therapies, Blood Rev. 29 (2015) 405-415. doi:10.1016/j.blre.2015.06.002.

[21] C. Guiter, I. Dusanter-Fourt, C. Copie-Bergman, M.L. Boulland, S. Le Gouvello, P. Gaulard, et al, Constitutive STAT6 activation in primary mediastinal large B-cell lymphoma, Blood. 104 (2004) 543-549. doi:10.1182/blood-2003-10-3545.

[22] L. Rui, N.C. Emre, M.J. Kruhlak, H.J. Chung, C. Steidl, G. Slack, et al., Cooperative epigenetic modulation by cancer amplicon genes, Cancer Cell. 18 (2010) 590-605. doi:10.1016/j.ccr.2010.11.013.

[23] M.A. Weniger, I. Melzner, C.K. Menz, S. Wegener, A.J. Bucur, K. Dorsch, et al., Mutations of the tumor suppressor gene SOCS-1 in classical Hodgkin lymphoma are frequent and associated with nuclear phospho-STAT5 accumulation, Oncogene. 25 (2006) 2679-2684. doi:10.1038/sj.onc.1209151.

[24] H. Dai, S. Ehrentraut, S. Nagel, S. Eberth, C. Pommerenke, W.G. Dirks, et al., Genomic landscape of primary medistinal B-cell lymphoma cell lines, PLoS One. 10 (2015) e0139663. doi:10.1371/journal.pone.0139663.

[25] J. Gunawardana, F.C. Chan, A. Telenius, B. Woolcock, R. Kridel, K.L. Tan, et al., Recurrent somatic mutations of PTPN1 in primary mediastinal B cell lymphoma and Hodgkin lymphoma, Nat Genet. 46 (2014) 329-335. doi:10.1038/ng.2900.

[26] V. Camus, A. Stamatoullas, S. Mareschal, P.J. Viailly, N. Sarafan-Vasseur, E. Bohers, et al., Detection and prognostic value of recurrent exportin 1 mutations in tumor and cell-free circulating DNA of patients with classical Hodgkin lymphoma, Haematologica. 101 (2016) 1094-1101. doi:10.3324/haematol.2016.145102. 
[27] I. García-Santisteban, I. Arregi, M. Alonso-Mariño, M.A. Urbaneja, J.J. Garcia-Vallejo, S. Bañuelos, et al., A cellular reporter to evaluate CRM1 nuclear export activity: functional analysis of the cancer-related mutant E571K, Cellular and Molecular Life Sciences. (2016) 1-15. doi:10.1007/s00018-016-2292-0.

[28] K. Mowen, M. David, Regulation of STAT1 Nuclear Export by Jak1, Mol Cell Biol. 20 (2000) 7273-7281.

[29] N.C. Reich, STATs get their move on, Jak-Stat. 2 (2013) e27080. doi:10.4161/jkst.27080.

[30] O. Ritz, K. Rommel, K. Dorsch, E. Kelsch, J. Melzner, M. Buck, et al., STAT6-mediated $\mathrm{BCL6}$ repression in primary mediastinal B-cell lymphoma (PMBL), Oncotarget. 4 (2013) 1093-1102. 


\section{Figure legends}

Fig. 1. STAT6 shuttles between the cytoplasm and the nucleus in IL4-stimulated 293-S6

\section{cells and in unstimulated PMBL cells}

(a) 293-S6 cultured cells were stimulated with IL4 $(10 \mathrm{ng} / \mathrm{ml})$. Cells were then harvested at the indicated times. Fractionated cytoplasmic and nuclear extracts were prepared from $10^{7}$ cells. Proteins were separated by SDS-PAGE, transferred onto nitrocellulose membranes and IB was done using Abs against STAT6 or p-Tyr641-STAT6 and then, as secondary Ab, a peroxidase-conjugated anti-rabbit IgG from abcam (ab6721). (b) 293-S6 cells were cultured on poly-L-lysine-coated coverslips and treated (or not for control) with IL4 ( $25 \mathrm{ng} / \mathrm{ml}$ ) for $1 \mathrm{~h}$. STAT6 and p-Tyr641-STAT6 were stained as described in the Methods section. Slides were counterstained with DAPI and then observed with a confocal microscope (x 180, magnification). Zoomed images $(x 6)$ of p-STAT6 staining from other fields. (c) Untreated PMBL cells were analysed for STAT6 and p-Tyr641-STAT6 localization by IF as described in (b).

Fig. 2. XPO1 and STAT6 colocalise in 293-S6 and PMBL cells

(a) 293-S6 cells were cultured on poly-L-lysine-coated coverslips and slides were processed for XPO1 (in red), STAT6 (in green), and DAPI staining as described previously.

Representative images are shown. In the merged image, the fluorescence intensity (in arbitrary units) of each fluorophore was estimated with the ImageJ software (available from https://imagej.nih.gov/il/) along a line (in yellow) crossing a representative cell. Data were exported from ImageJ to generate the curves of fluorescence intensity (in arbitrary units, $\mathrm{AU}$ ) as a function of the distance (left to right, in $\mathrm{nm}$ ). The experiment has been carried out twice. (b) MedB1 and U2940 PMBL cells were cytospun and stained as described previously. The fluorescence intensity of each fluorophore was estimated along a line crossing one or 
two representative cell(s) in the merged image and reported on the graphs. Representative images are shown. The experiment has been carried out three times.

\section{Fig. 3. XPO1 interacts with STAT6}

The Duolink ${ }^{\circledR}$ PLA was used on 293-S6, U2940 (wt/wt) and MedB1 (wt ampl./E571K) cells to investigate XPO1/STAT6 interactions. Slides were incubated with the primary Abs except for the negative control (Ctrl) and then with the secondary Abs conjugated to the MINUS and PLUS probes. The ligation and amplification steps were next performed. Slides were counterstained with DAPI and then observed with the confocal microscope ( $x 180$, magnification). Each red dot (some are arrowed in white) is representative of one proteinprotein interaction. Representative images from three independent experiments are shown. On the right are zoomed images $(x 3)$ from the corresponding white squared fields.

\section{Fig. 4. STAT6 is a cargo of XPO1}

(a) PMBL cells were seeded at a density of $3 \times 10^{5}$ cells/ well in 96-well plates and treated with the indicated concentrations of KPT-330 (0.1-20 nM). Cell viability was analysed using an MTS assay (CellTiter 96®AQueous One Solution Cell Proliferation Assay, Promega). The mean of the background (absorbance of three wells with no cells) was deduced from each absorbance (OD at $490 \mathrm{nM}$ ) value of each clone treated with the drug. For each set of culture conditions, the means \pm SD of triplicate values are indicated on the graph. The experiment has been carried out twice with similar results. (b) Cells were left untreated (nt) or treated with $1 \mu \mathrm{M}$ KPT-330 for $4 \mathrm{~h}$ before STAT6 and XPO1 staining and IF analysis as described previously. Representative images are shown.

\section{Fig. 5. Structural similarities and differences for STAT proteins}

The functional domains of STAT1, STAT3 and STAT6 proteins are schematized. Abbreviations : $\mathrm{N}$, terminal domain involved in dimerization; $\mathrm{CC}$, coiled-coil interacting domain; DNA, DNA 
binding domain; LK, linker domain; $\mathrm{SH}$, SH2 dimerization domain; $\mathrm{Y}$, phosphorylated tyrosine; TA, transactivation domain; NES, nuclear export signal. 
Table 1. Mutational status of XPO1 and STAT6 in PMBL cell lines

\begin{tabular}{|l|l|l|}
\hline Cell lines & XPO1 $^{\text {a }}$ & STAT6 \\
\hline K1106 & wt ampl./wt & wt $^{\text {b }}$ \\
\hline MedB1 & wt ampl./E571K & wt/N417Y and N430T \\
\hline U2940 & wt/wt & wt $^{\mathrm{c}}$ \\
\hline
\end{tabular}

Data were obtained from ${ }^{\mathrm{a}}[5],{ }^{\mathrm{b}}[2],{ }^{\mathrm{c}}[30]$. Abbreviations: wt, wild-type; ampl., amplified. 
Table 2. Putative NES domains in STAT6

\begin{tabular}{|l|l|l|l|}
\hline Protein name & Position & Sequence & Score \\
\hline STAT6_HUMAN & $105-119$ & GEKKAVMEQFRHLPM & 0.047 \\
\hline STAT6_HUMAN & $130-144$ & FKTGLRRLQHRVGEI & 0.020 \\
\hline STAT6_HUMAN & $132-146$ & TGLRRLQHRVGEIHL & 0.080 \\
\hline STAT6_HUMAN & $\mathbf{1 8 4 - 1 9 8}$ & QETTGELEAAKALVL & $\mathbf{0 . 7 5 0}$ \\
\hline STAT6_HUMAN & $189-203$ & ELEAAKALVLKRIQI & 0.205 \\
\hline STAT6_HUMAN & $\mathbf{3 9 4 - 4 0 8}$ & LGPGKLPIQLQALSL & $\mathbf{0 . 5 4 8}$ \\
\hline STAT6_HUMAN & $396-410$ & PGKLPIQLQALSLPL & 0.432 \\
\hline STAT6_HUMAN & $398-412$ & KLPIQLQALSLPLVV & 0.086 \\
\hline STAT6_HUMAN & $399-413$ & LPIQLQALSLPLVVI & 0.121 \\
\hline STAT6_HUMAN & $400-414$ & PIQLQALSLPLVVIV & 0.052 \\
\hline STAT6_HUMAN & $649-663$ & TVERDQPLPTPELQM & 0.175 \\
\hline STAT6_HUMAN & $720-734$ & EPHLQMPPSLGQMSL & 0.178 \\
\hline STAT6_HUMAN & $791-805$ & FPPLLPPTEQDLTKL & 0.129 \\
\hline STAT6_HUMAN & $793-807$ & PLLPPTEQDLTKLLL & 0.144 \\
\hline STAT6_HUMAN & $827-841$ & SHYGQSGISMSHMDL & 0.059 \\
\hline
\end{tabular}

Data were obtained with the LocNES computational tool

(http://prodata.swmed.edu/LocNES/LocNES.php). The two sequences in bold, having a high confidence score were identified as putative NES. 


\section{Highlights}

- STAT6 is a cargo of XPO1

- Selinexor inhibits STAT6 nuclear export

- The E571K XPO1 recurrent mutation has no impact on STAT6 nuclear export

- XPO1 and STAT6 are potent targets for PMBL therapy 


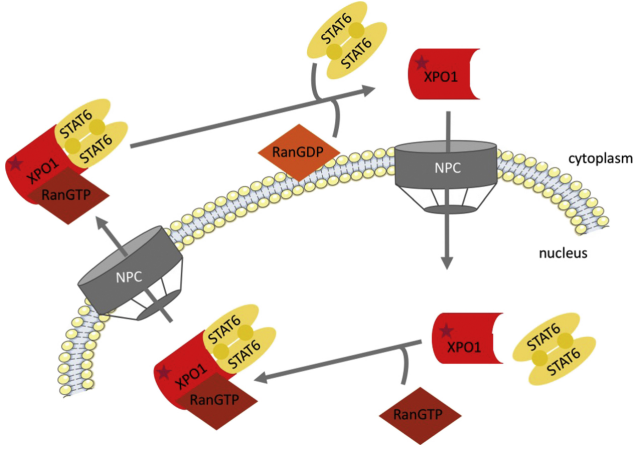

Graphics Abstract 


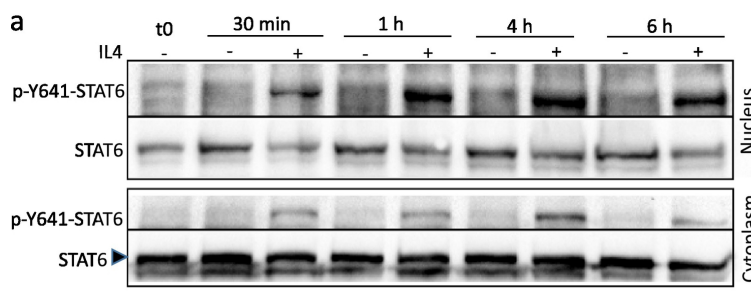

b

293-S6

C
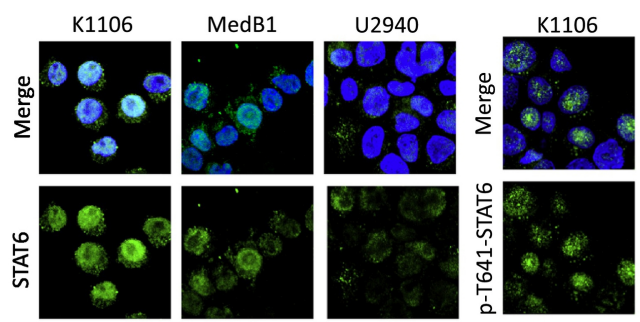

음

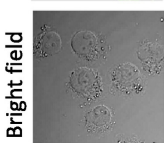

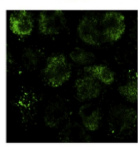
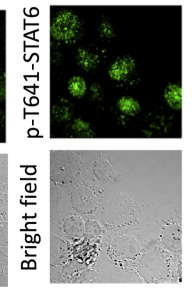

MedB1
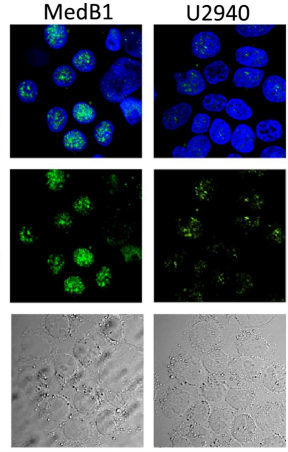

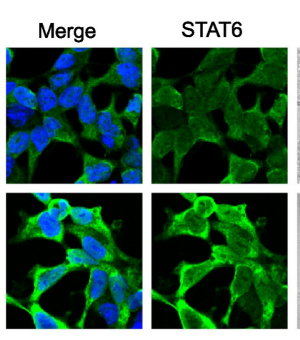

Bright field

- IL4
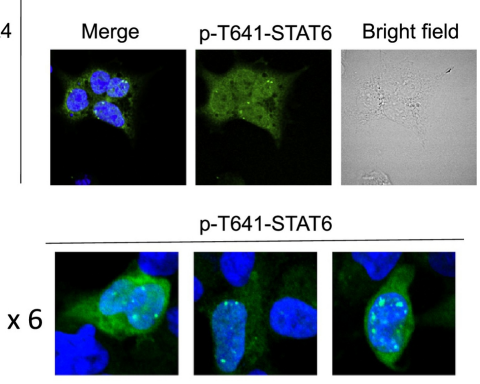

Figure 1 


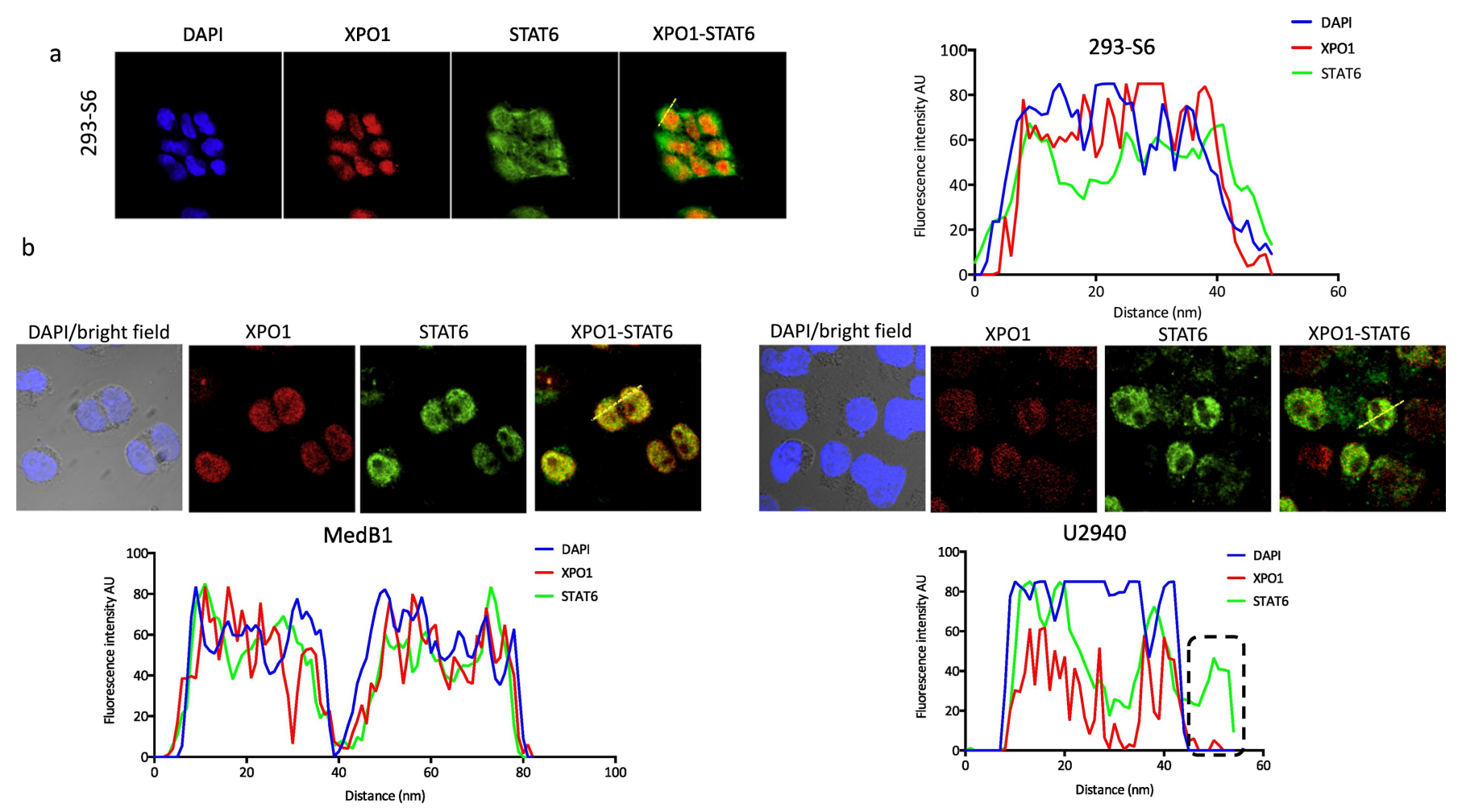

Figure 2 


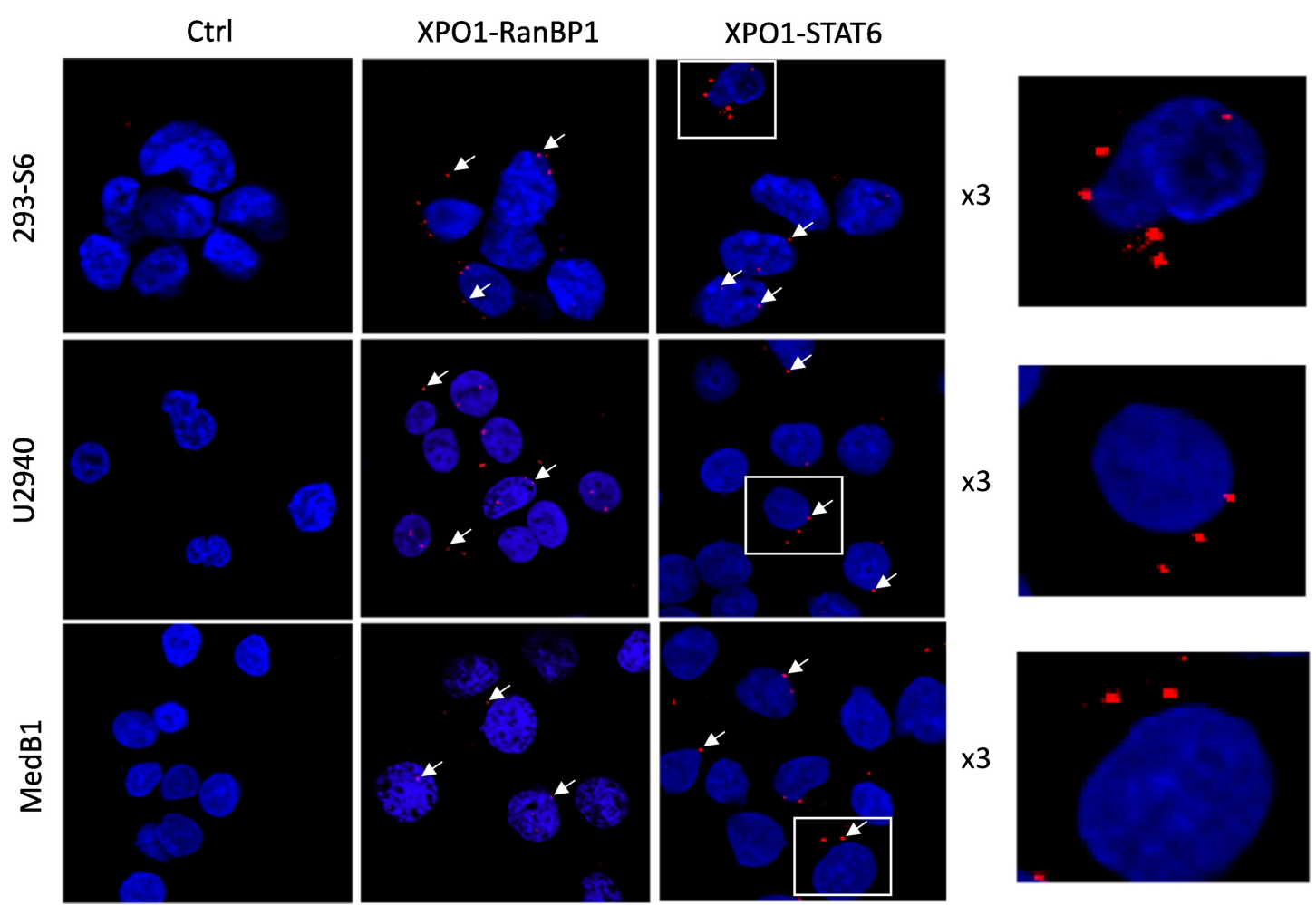

Figure 3 

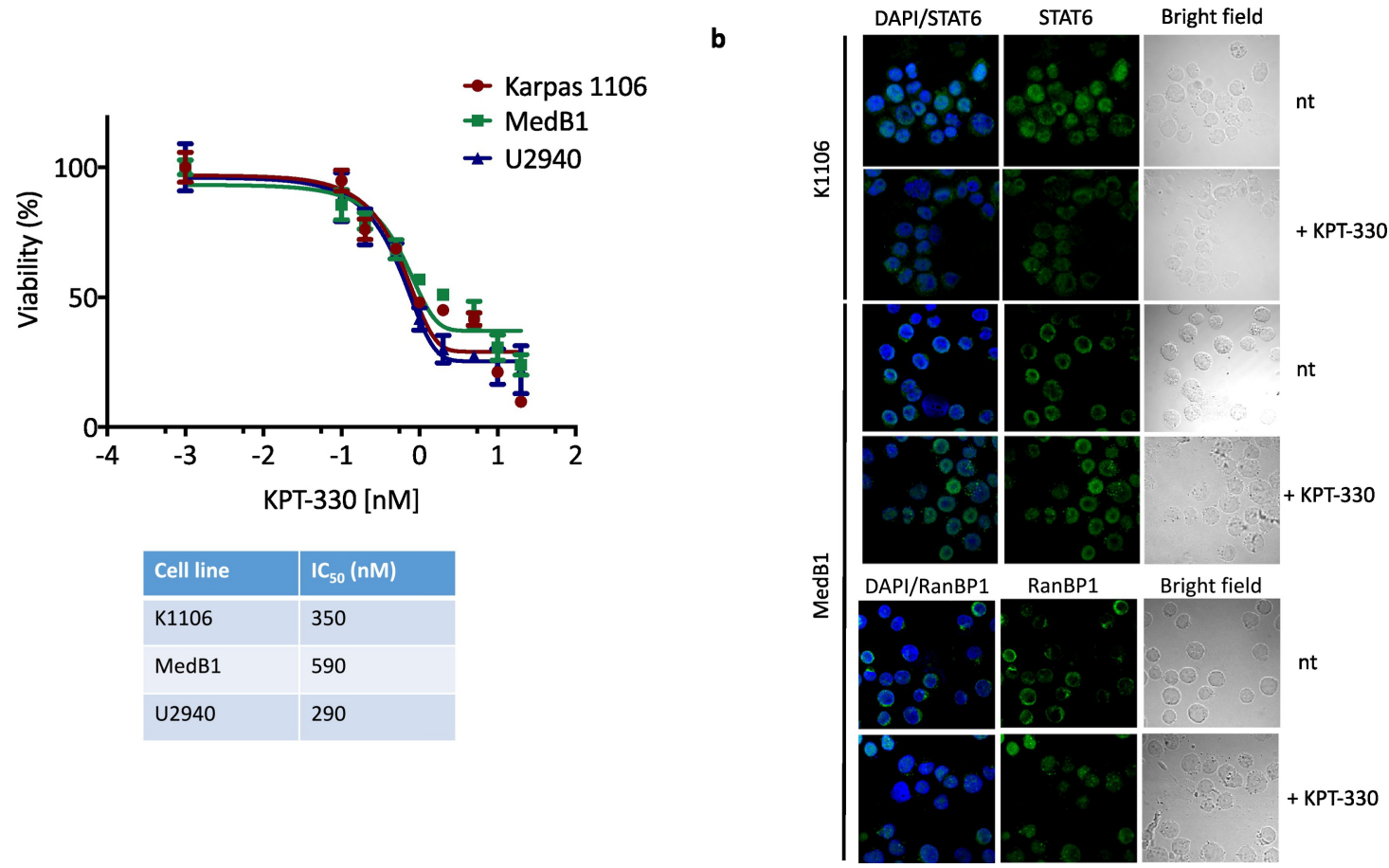


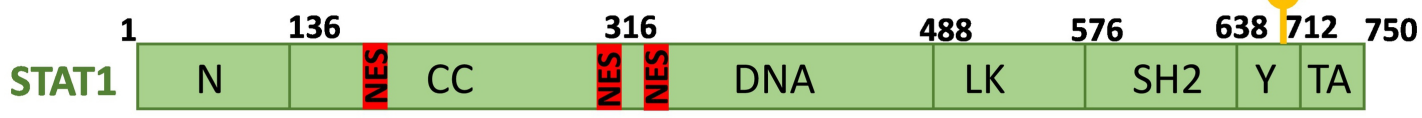

197-205 302-320 399-410

705
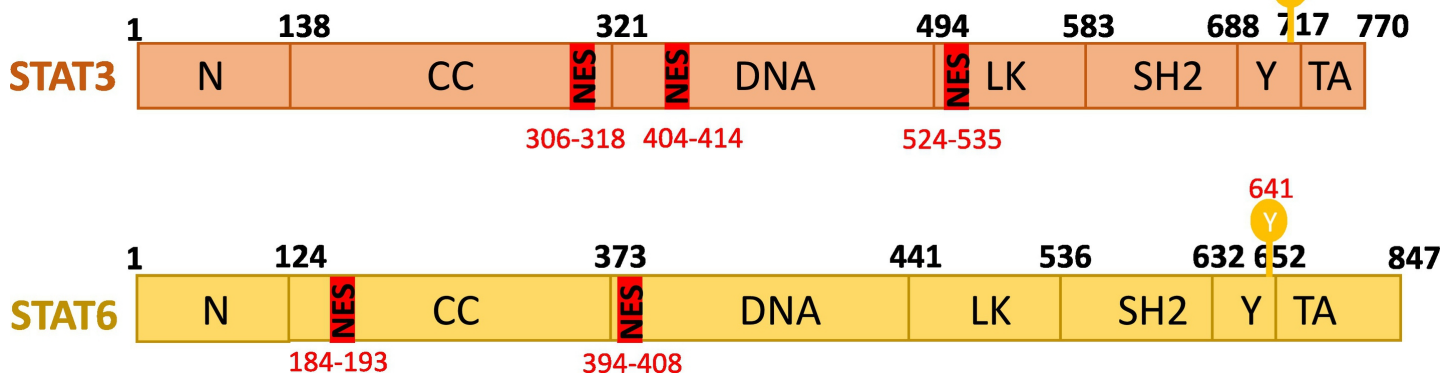

Figure 5 